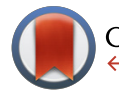

CrossMark \&lick for updates

Cite this: Lab Chip, 2015, 15, 3495

Received 8th May 2015

Accepted 9th July 2015

DOI: $10.1039 / c 51 c 00523 j$

www.rsc.org/loc

\title{
Direct coupling of a free-flow isotachophoresis (FFITP) device with electrospray ionization mass spectrometry (ESI-MS)
}

\author{
J. K. Park, ${ }^{a}$ C. D. M Campos, ${ }^{\text {ab }}$ P. Neužil, ${ }^{\text {ac }}$ L. Abelmann, ${ }^{\text {ad }}$ R. M. Guijt ${ }^{\text {ae }}$ and A. Manz ${ }^{\star a}$
}

We present the online coupling of a free-flow isotachophoresis (FFITP) device to an electrospray ionization mass spectrometer (ESI-MS) for continuous analysis without extensive sample preparation. Free-flowelectrophoresis techniques are used for continuous electrophoretic separations using an electric field applied perpendicular to the buffer and sample flow, with FFITP using a discontinuous electrolyte system to concurrently focus a target analyte and remove interferences. The online coupling of FFITP to ESI-MS decouples the separation and detection timeframe because the electrophoretic separation takes place perpendicular to the flow direction, which can be beneficial for monitoring (bio)chemical changes and/or extensive MS $^{n}$ studies. We demonstrated the coupling of FFITP with ESI-MS for simultaneous concentration of target analytes and sample clean-up. Furthermore, we show hydrodynamic control of the fluidic fraction injected into the MS, allowing for fluidically controlled scanning of the ITP window. Future applications of this approach are expected in monitoring biochemical changes and proteomics.

\section{Introduction}

Biochemical pathways are complex and typically involve a wide range of compounds covering a wide dynamic range. The elucidation of these pathways and their control mechanisms requires sophisticated analytical methods, typically removing interferences and enhancing the concentration of targets to allow for their detection. High resolution analytical techniques such as high performance liquid chromatography (HPLC) and capillary electrophoresis (CE) are often coupled with mass spectrometry to obtain information about the amount and identity of these compounds. ${ }^{1}$

Electrophoretic techniques separate analytes based on differences in their migration velocity in an applied electric field. ${ }^{2,3}$ Isotachophoresis (ITP) is a mode of electrophoresis, where the sample is placed in a discontinuous electrolyte system, comprised of a leading and trailing electrolyte (LE and TE, respectively). The LE is selected to contain ions with mobility higher than that of the target analytes, while the TE is selected to contain ions with mobility lower than the target

\footnotetext{
${ }^{a}$ Korea Institute of Science and Technology (KIST)-Europe, Campus e 7 1, 66123, Germany. E-mail: manz@kist-europe.de

${ }^{b}$ State University of Campinas, PO BOX 6154, 13083-970, Brazil

${ }^{c}$ Central European Institute of Technology, Brno University of Technology, CZ-616 00, Brno, Czech Republic

${ }^{d}$ MESA+, University of Twente, Enschede, The Netherlands

${ }^{e}$ School of Medicine and ACROSS, University of Tasmania, Private Bag 26 Hobart TAS 7001, Australia
}

analytes. The ITP window covers the mobility range between the LE and TE. The Kohlrausch regulating value,

$$
\omega=\sum \frac{C_{\mathrm{i}} Z_{\mathrm{i}}}{\left|\mu_{\mathrm{i}}\right|}
$$

remains constant because ions arrange according to their electrophoretic mobility $\left(\mu_{\mathrm{i}}\right)$ and charge $\left(z_{\mathrm{i}}\right)$ by regulating their concentration $\left(c_{\mathrm{i}}\right)$. As this affects the current density, the electric field strength $(E)$ in each zone is adjusted so that all zones move at an equal velocity, $v_{\mathrm{i}}=\mu_{\mathrm{i}} E^{4,5}$

When dealing with samples containing compounds across a wide dynamic range in zone electrophoresis, analytes/interferences in high concentration broaden due to ion diffusion following Fick's law. In ITP, compounds outside the separation window dissipate in the LE or TE. Concentration differences within the window are evened out because high concentration compounds are diluted by lengthening their zone, low concentration compounds are concentrated by narrowing the zone. This facilitates the handing of samples across a wide dynamic range making it a very attractive technique for studying biochemical processes with high complexity in chemical diversity and dynamic range. Additionally, ions that diffuse into a higher (or lower) mobility zone develop a lower (or higher) velocity in the lower (or higher) electric field and migrate back into their original zone. This is known as the self-focusing effect. ITP has been extensively used in 
capillaries and on microchips, as discussed in various review articles. $^{6,7}$

Dictated by eqn (1), the length of the ITP band increases with the amount of analyte once the target concentration has been reached. This complicates analyte identification based on migration time and has limited the popularity of ITP as an analytical technique. At low analyte concentrations, the concentration required to achieve plateau mode cannot be achieved and analytes stack together in a narrow zone between the $\mathrm{LE}$ and $\mathrm{TE}$, a phenomenon referred to as peak mode ITP. In peak mode, concentration factors of 10000 to a million fold have been achieved. ${ }^{8-10}$

Analytical separations are typically conducted through the injection and separation of a discrete sample volume in a batch-mode process. The separated zones sequentially achieve the detector, triggering a response for a short period of time. The sampling interval is typically determined by the analysis time. Free-flow electrophoresis (FFE) comprises a group of continuous separation techniques that, whilst often employed for purification, can also be used analytically. The separation field is applied perpendicular to the flow of buffer and sample. The first FFE device was designed at relatively large scale, where the depth was in the range of millimetres. ${ }^{11}$ Since 1994 several microfluidic devices have been developed. Miniaturising the FFE device reduces the amount of sample required for analysis. It also limits the Joule heating problem by improving dissipation and increasing electrical resistance, allowing for the use of increased field strengths and hence higher resolution separations. ${ }^{12-21}$ Coupling of a FFE device to an electrospray ionization mass spectrometer (ESI-MS) allows for decoupling of the detection and separation timeframes and was beneficial for reaction monitoring purposes in zone electrophoresis mode. ${ }^{22,23}$

Whilst FFE is typically conduced as zone electrophoresis in a continuous electrolyte system, the use of a discontinuous electrolyte system allows for FFITP. ${ }^{24}$ Detection in FFITP is a challenging task, with optical detection following fluorescent labelling of the samples being the most commonly used method. Optical detection, however, is complicated in ITP because the separated analyte zones are stacked next to each other, requiring the use of spacers. ${ }^{25}$

Here, we demonstrate the online coupling of an FFITP device with ESI-MS, enabling continuous analyte concentration and clean-up before injection into the MS, as illustrated in Fig. 1. Hydrodynamic flow control was used to direct zones of interest into the MS, and enabled scanning of the ITP system. As mentioned before, analyte identification in analytical ITP with universal detection is complicated by the changes in migration time with sample composition. Connection with an MS eliminates this issue by allowing for the identification of the analyte based on its mass. In plateau mode, the hydrodynamically controlled scanning also provides a measure for the zone width, and hence analyte concentration. The proposed method is ideally suited for proteomic and metabolic studies, where the FFITP can simultaneously concentrate trace analytes in a specific mobility range in peak mode ITP,

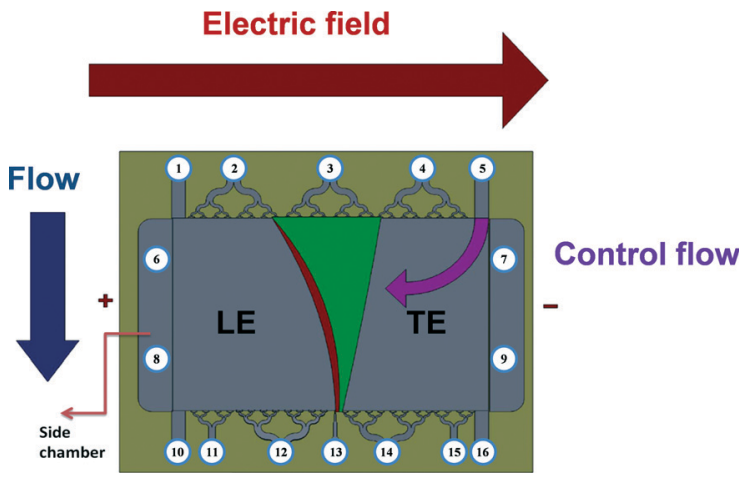

Fig. 1 Principle of operation of the FFITP chip. By applying an electric field perpendicular to the flow direction, the target analytes are focused between the leading (LE) and terminating buffer (TE). Increasing the flow at inlet 5 will shift the stream to a desired outlet (13).

whilst removing interferences with mobilities outside the ITP window. The concentrated targets are then continuously directed into the MS, providing its resolving power to identify and analyse the concentrated analytes in a timeframe independent of the analytical separation.

\section{Model}

To support the design and experiments, we simulated the flow patterns in the chip, as well as the concentration and purification profiles during isotachophoresis.

\section{Fluidic}

One of the key features of the chip design is the possibility to collect specific fractions at one of the outlets. This can be controlled by the flow rate at the inlets, so that the ratio of the flow changes. ${ }^{22,26,27}$ In order to achieve the exact relationship of the flow ratio change and the shifted streamline, simulations were conducted using FreeFEM $++{ }^{28}$ simulation software using a slight simplification of the design (Fig. 2A).

The Stokes differential equation for an incompressible Newtonian fluid was solved, with boundary conditions set by flow velocities in both directions at all surfaces. Grid resolution was tested by decreasing the grid size by a factor of two, accepting the resolution if the difference in flow shift was less than 5\%. An example of an obtained flow pattern is shown in Fig. 2A. In Fig. 2B, the $x$-component of the velocity is given for a change in flow rate at inlet 1 from 5 to $6 \mu \mathrm{L}$ $\min ^{-1}$ by $0.1 \mu \mathrm{L} \min ^{-1}$ increments, reducing the flow rate

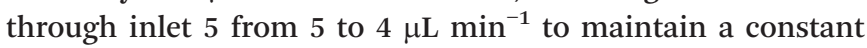
total flow rate of $10 \mu \mathrm{L} \mathrm{min}{ }^{-1}$. The outlet position for the sample stream line is defined at the point where the $x$-component of the velocity is zero, and shows a trend towards the right with an increase in flow rate from inlet 1.

\section{Isotachophoresis}

In capillary and free flow ITP, conductivity and optical detection are most frequently used, but this method can also be 
A

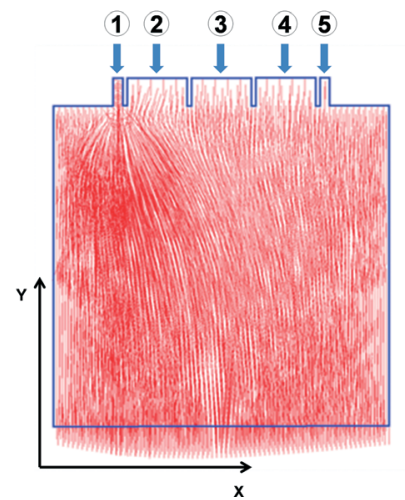

B

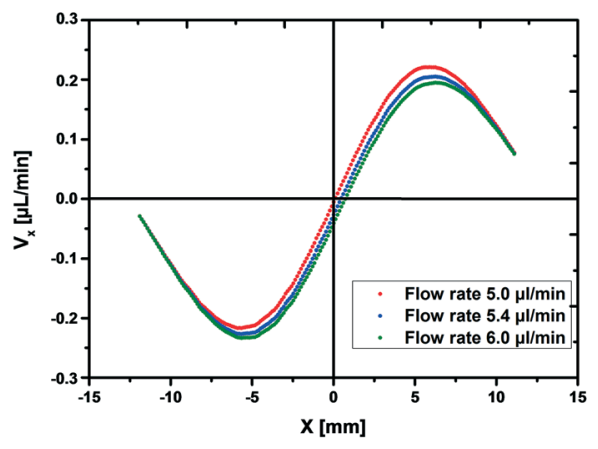

C

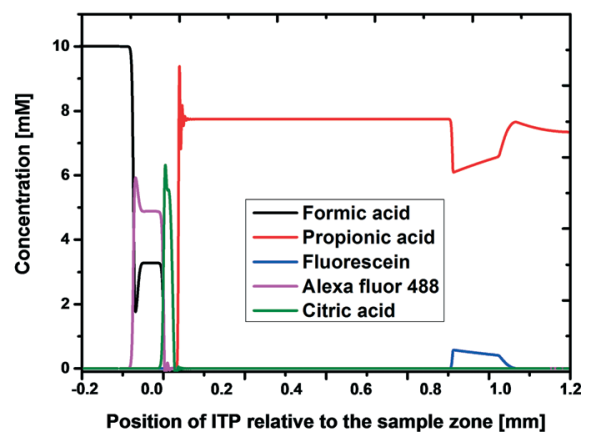

Fig. 2 (A) Simplified drawing of the device showing the velocity vectors. For shifting the stream, the flow rate at inlet 1 was increased. (B) The $x$-component of the velocity at the outlet of the device as function of the flow at inlet 1 . The point at which the $x$-component becomes zero shifts towards the right with increasing flow, by increasing the flow rate with a $0.1 \mu \mathrm{L} \mathrm{min}{ }^{-1}$ step. (C) Result of the ITP simulation, showing Alexa Fluor 488 and citric acid being concentrated in the ITP window while fluorescein dissipates into the TE. The ITP system is depicted right to left with the relative position of the window positioned at 0 , LE at negative values and TE at the positive values.

used in conjunction with a mass spectrometer. The limited range of ESI-MS compatible buffers, however, restricts the range of LEs and TEs that can be used. Gebauer et al. ${ }^{29}$ recently proposed manipulating the ITP window by adjusting the $\mathrm{pH}$ and therefore effective mobility of the LE and TE. Here, a similar approach was followed, and resulted in the selection of formic acid as the leader and propionic acid as the terminator. With the LE adjusted to $\mathrm{pH} 4.3$ using $\mathrm{NH}_{4} \mathrm{OH}$; the resulting effective mobilities are shown in Table $1 .^{30}$ The ITP process was modeled using SIMUL ${ }^{31}$ in constant current mode $(-1.125 \mu \mathrm{A})$. The LE was $10 \mathrm{mM}$ formic acid adjusted to $\mathrm{pH} 4.3$ with $8 \mathrm{mM}$ ammonium, and the TE was $7 \mathrm{mM}$ propionic acid with a pH of 3.5. Alexa Fluor $488(3 \mathrm{mM})$, citric acid $(1 \mathrm{mM})$, fluorescein $(2 \mathrm{mM})$ and glycolic acid $(1 \mathrm{mM})$ were used as model analytes, with their effective mobilities given in Table 1. The SIMUL results are presented in Fig. 2C. To maintain continuity in the figures throughout the manuscript, the LE is on the left, TE on the right hand side. The ITP window defined between the LE (formic acid) and TE (propionic acid). The target analytes stack in the window in order of decreasing mobility, with the fast Alexa Fluor 488 adjacent to the LE followed by Alexa Fluor 488. Fluorescein, the model contaminant, has mobility lower than the TE and therefore dissipated from the ITP window into the TE zone.

Table 1 Effective mobilities in the established ITP system determined by SIMUL

\begin{tabular}{llll}
\hline Compound & $\mathrm{p} K_{\mathrm{a}}$ & $\mu\left(10^{-9} \mathrm{~m} \mathrm{~V}^{-1} \mathrm{~s}^{-1}\right)$ & $\begin{array}{l}\mu_{\text {eff }} \\
\left(10^{-9} \mathrm{~m} \mathrm{~V}^{-1} \mathrm{~s}^{-1}\right)\end{array}$ \\
\hline Alexa Fluor 488 & - & & 36 \\
Fluorescein & $6.8 ; 4.4$ & $35.9,19$ & 0.5 \\
Citric acid & $6.41 ; 4.76 ; 3.13$ & $74.4 ; 54.7 ; 28.7$ & 28.9 \\
Glycolic acid & 3.89 & 42.4 & 27.1 \\
Formic acid & 3.75 & 56.6 & 44.3 \\
Propionic acid & 4.87 & 37.1 & 1.6
\end{tabular}

\section{Experimental}

\section{Chemicals}

As LE, we used $10 \mathrm{mM}$ Formic acid adjusted to $\mathrm{pH} 4.29$ with ammonium hydroxide, $7 \mathrm{mM}$ propionic acid ( $\mathrm{pH}$ 3.55) was used as TE. For the MS scanning study, samples contained 1 $\mathrm{mM}$ fluorescein, $1 \mathrm{mM}$ citric acid, $1 \mathrm{mM}$ Alexa Fluor 488, and $1 \mathrm{mM}$ glycolic acid. All chemicals were purchased from Sigma Aldrich (Germany) with the exception of Alexa Fluor 488, which was purchased from Life Technology (Germany).

\section{Layout}

The layout of the device is shown in Fig. 1. The device consisted of five inlets (1-5), a $23 \mathrm{~mm} \times 15 \mathrm{~mm}$ separation chamber, two side chambers for connecting the electrodes (6-9) and seven outlets (10-16). The three middle inlets were equipped with binary tree structures to evenly distribute the input solutions into the chamber. The outer two inlets ( 1 \& 5) were designed for hydrodynamic control. All outlets were designed similarly to the inlets except for the middle outlet (13), which has a narrower $(100 \mu \mathrm{m}$ wide) outlet channel for sample collection. The electrode chambers are connected to the main chamber using an array of $25 \mu \mathrm{m}$ wide channels, 50 $\mu \mathrm{m}$ apart. Arrays of pillars were introduced to strengthen the support of the chamber wall (Fig. 3A). The voltages were applied to the buffer-filled reservoirs to minimize the $\mathrm{pH}$ changes inside the chamber. Gas bubbles, generated by electrolysis, were prevented from entering the main chamber by reservoirs and an array of channels in the side chamber. Fig. 3B shows the side reservoir and the connectors, which are used for connecting the tubing. To provide fluidic access by Teflon tubing (ID: $0.5 \mathrm{~mm}$, OD: $1.6 \mathrm{~mm}$, ProLiquid GmbH, Germany) to the chip, bootlace ferrules (OD: $1 \mathrm{~mm}$, height: $8 \mathrm{~mm}$, Bauhaus, Germany) were bonded to inlets 1-4 and outlets 10-12 and 14-16 using epoxy (UHU, Germany). Outlet 13 was connected using a One-Piece Fitting \& Bonded-Port 
A

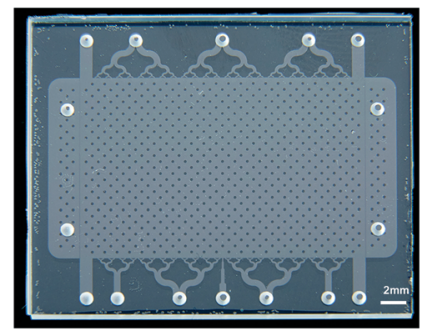

B

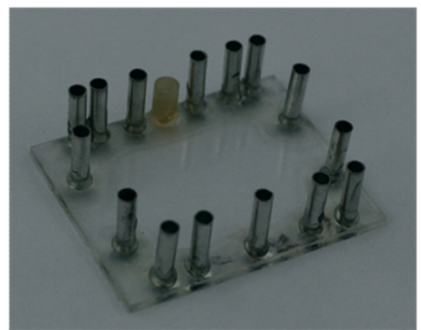

(C)

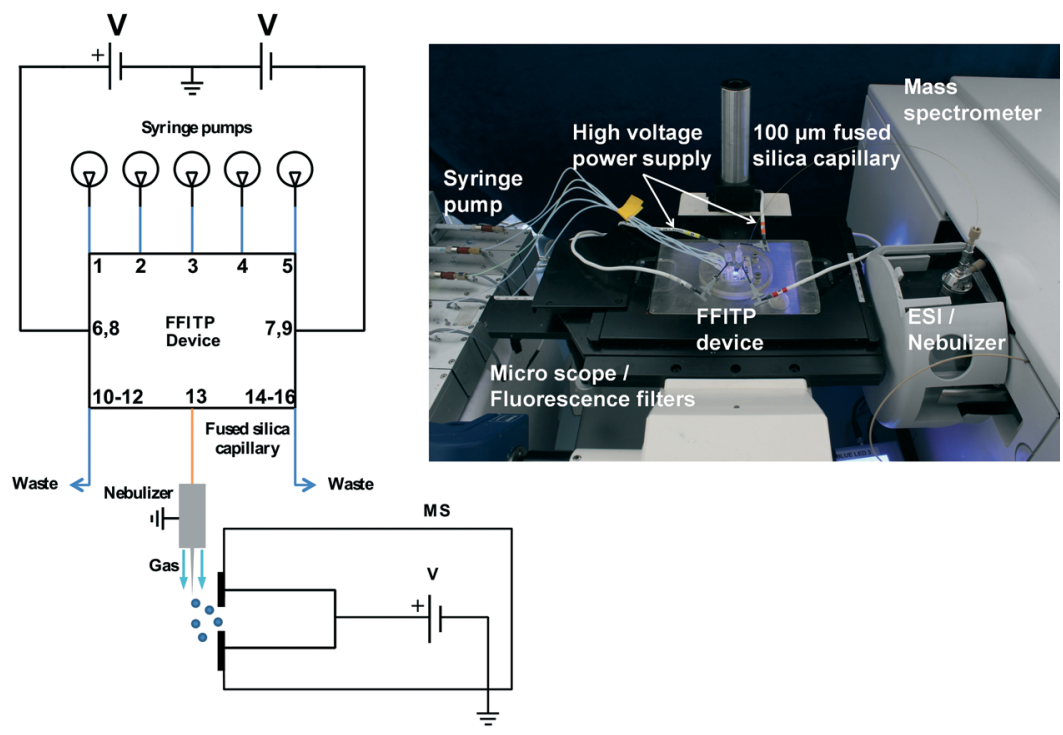

Fig. 3 (A) Photograph of the device implementation in glass. The side chambers are separated from the main chamber by $25 \mu \mathrm{m}$ wide grooves. Pillars were introduced to avoid collapse during thermal bonding and to prevent breaking by the high back pressure inside the chamber. (B) Connections for the tubing and the side reservoirs which are used to connect the electrodes. (C) Connection of the FFITP chip to the MS with a diagram showing the connection points for the electric field, syringe pumps and ESI-MS. The chip was mounted on an inverted optical microscope (photograph).

Connector (Labsmith, USA). The metal bootlace ferules were also mounted to the four electrode outlets (6-9) to act as buffer reservoirs.

\section{Fabrication}

The starting substrate was a $500 \mu \mathrm{m}$ borosilicate glass wafer. After cleaning in $\mathrm{HNO}_{3}$ and a quick dump rinse in de-ionized (DI) water, a $5 \mathrm{~nm} \mathrm{Cr}$ adhesion layer, and $150 \mathrm{~nm}$ Au layer were deposited by magnetron sputtering. The location of channels was defined in the metal layers by optical lithography using a $1.7 \mu \mathrm{m}$ thick OLIN Oir 907-17 positive photoresist and subsequent wet etched in a mixture of $\mathrm{KI}: \mathrm{I}_{2}: \mathrm{DI}$ water $(4: 1: 40)$ to remove the $\mathrm{Au}$, and a dedicated Chromium etchant. The separate etching steps were repeated to ensure that all metal was removed. Quick dump rinses were performed between the etching steps. Using the metal mask for protection, the channels were etched into the glass wafer using a $\mathrm{HF}(25 \%) / \mathrm{HCl}(2.5 \%) / \mathrm{DI}$ water mixture. Etching was performed in steps, monitored by surface profilometry, until the depth was $5.0 \pm 0.1 \mu \mathrm{m}$. The etchant was diluted during the last steps. Through holes were defined in a laminated Ordyl BF 410 dry resist foil. The holes were defined by powder blasting with $30 \mu \mathrm{m} \mathrm{Al} \mathrm{O}_{3}$ particles, using a pressure of 4.6 bars, massflow of $3-12 \mathrm{~g} \mathrm{~min}^{-1}$, leading to an etch rate of approximately $91 \mu \mathrm{m} \mathrm{g}^{-1} \mathrm{~cm}^{-2}$. The foil was removed in $\mathrm{Na}_{2} \mathrm{CO}_{3}$ solution and the wafers where thoroughly rinsed to remove all particles. The remaining $\mathrm{Au} / \mathrm{Cr}$ layer was stripped using the same etching procedure as before. The resulting wafer and a support wafer were cleaned, and dipped into a
$25 \% \mathrm{KOH}$ solution to obtain a good temporary bond (prebond). The channels were defined by bonding both wafers together, using a 60 second high pressure prebonding step in an EV620 mask-aligner at $340{ }^{\circ} \mathrm{C}$ and a one hour postbond in an oven at $600-650{ }^{\circ} \mathrm{C}$ under atmospheric conditions. Chips were protected by laminated foils on both sides and diced into individual devices by an NL-CLR- Disco DAD diamond dicing saw.

\section{Experimental setup}

The setup of the experiment is shown in Fig. 3C. The FFITP device was mounted on an Axiovert 100 (Zeiss GmbH, Germany) inverted microscope. LEDs (type M470L3, Thorlabs GmbH, Germany) with a $470 \mathrm{~nm}$ principal wavelength and maximum optical power of $650 \mathrm{~mW}$ were used for sample illumination. All fluorescence imaging and measurement experiments were done using a 470/525 ex/em filter set (model 49002, Chroma Technology Corp. USA). Imaging was performed using a $5 \times$ objective lens (Zeiss $\mathrm{GmbH}$, Germany) and a color CCD camera model C5 (Jenoptik GmbH, Germany) with the LED intensity adjusted to $1.6 \mathrm{~A}$. The fluorescence intensity measurements were performed using a single point detector made using a 50× objective lens and photomultiplier tube (PMT) (model H10722-01, Hamamatsu Photonics, Co., Germany) with the gain set by a $0.5 \mathrm{~V}$ bias. Samples and buffer were injected through a neMESYS Low Pressure Syringe Pump system comprised of five syringe modules (Cetoni GmbH, Germany). To connect the tubing from the syringes to the device, bootlace ferrules were bonded to the 
glass chip using epoxy (UHU, Germany), shown in Fig. 3B. The potential difference was applied using a HVS448 High Voltage Sequencer (Labsmith, USA).

\section{Interface from chip to MS}

A fused silica capillary with an outer diameter of $360 \mu \mathrm{m}$, inner diameter of $100 \mu \mathrm{m}$ and length of $30 \mathrm{~cm}$ was used to connect the FFITP chip to the ESI interface of the $1100 \mathrm{LC} /$ MSD mass spectrometer (Agilent, Germany). A commercially available connector (One-Piece Fitting \& Bonded-Port Connector, Labsmith, USA) was used to connect the fused silica capillary to the chip. A 1/32" OD PEEK tubing Sleeve (IDEX, USA) was used to guide the capillary into the ESI interface of the 1100 LC/MSD mass spectrometer (Agilent, Germany). The mass spectrometer was operated in negative mode with a potential of $4000 \mathrm{~V}$, fragmentation factor 100, and nitrogen was used as nebulizing gas. No sheath flow was used. The flow rate from the chip outlet through the capillary was 1.5 $\mu \mathrm{L} \min ^{-1}$ as determined by collection and weighing at 10 minute intervals when the free flow device operated at $2 \mu \mathrm{L}$ $\min ^{-1}$. At this flow rate, there is a 2 minute delay between the analytes leaving the device and entering the MS.

\section{Experimental procedure}

The FFITP device was first filled with LE (inlets 1 and 2) and TE (inlets 4 and 5). Once the main chamber and electrode reservoirs were filled, the reservoirs at the side chambers were sealed using the Platinum electrodes. Then the sample was introduced at inlet 3 , and after equilibration of the flows, the electric field was applied across the chamber.

\section{Result and discussion}

Control of the focused stream

The measured displacement is plotted as a function of the flow rate change at inlet 1 for a total flow rate of $10 \mu \mathrm{L} \mathrm{min}$ m $^{-1}$ in Fig. 4 using black dots, demonstrating good agreement with the simulated results (indicated with red line). A linear relationship was found between the flow rate and displacement, with a $0.1 \mu \mathrm{L} \mathrm{min}{ }^{-1}$ increment resulting in a $30 \mu \mathrm{m}$ shift. After a flow rate change at the inlet, approximately 45 seconds were required for the outlet flow to stabilize at its new position. Inserts 1 and 2 in Fig. 4 are microscope images taken at $5.0 \mu \mathrm{L}$ $\min ^{-1}$ and $6.0 \mu \mathrm{L} \mathrm{min}{ }^{-1}$, again taken at a total flow rate of $10 \mu \mathrm{L} \mathrm{min}{ }^{-1}$, to illustrate the shift of the focused stream line.

\section{Isotachophoresis}

The main advantage of ITP over zone electrophoresis is that clean-up and concentration of trace analytes can be achieved simultaneously through the selection of the LE and TE. To demonstrate the FFITP-MS, model compounds were selected because they could be visualized using fluorescence microscopy and/or determined by MS. Fluorescein was used as a model contaminant with a lower mobility than the TE and the targets. Citric acid was selected as target analyte because

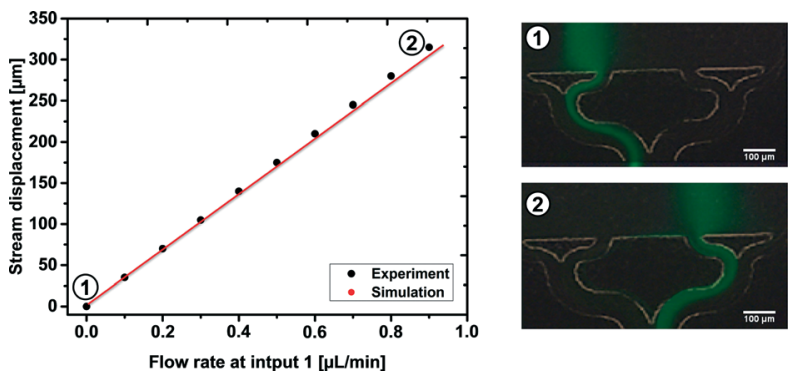

Fig. 4 Comparison of the experimental data and the simulation of the hydrodynamic control of the focused stream line. Indicated on the vertical axes is the $x$-position the outlet at which the $x$-component of the velocity is zero. This point, at which the liquid no longer displaces in $x$-direction, is shifting to the left side with increasing flow at input 1.

its electrophoretic mobility is similar to that of Alexa Fluor 488, but unlike Alexa Fluor 488 it yields a response in the MS. This allows the visualization of the ITP window using Alexa Fluor 488, and the analysis of the effluent by MS.

In order to determine the optimal separation voltage, the fluorescence intensity of the Alexa Fluor 488 zone was studied as a function of the potential difference applied across the FFITP device with a constant flow rate of $2 \mu \mathrm{L} \mathrm{min}^{-1}$ (Fig. 5A).

The intensity increased with the applied potential difference, leveling off around $1200 \mathrm{~V}\left(E=520 \mathrm{~V} \mathrm{~cm}^{-1}, I=100 \mu \mathrm{A}\right)$, indicating that steady state was achieved. When $2000 \mathrm{~V}$ was applied across the device $\left(E=870 \mathrm{~V} \mathrm{~cm}^{-1}, I=200 \mu \mathrm{A}\right)$, bubble generation was observed, indicating excessive Joule heating and/or electrolysis at the electrodes.

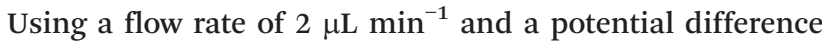
of $1200 \mathrm{~V}$, a sample comprising of fluorescein, Alexa Fluor 488 and citric acid was loaded. The microscope image in Fig. 5B confirms the validity of the selected electrolyte system. The fluorescein is effectively removed from the sample, demonstrated by a faint zone representing the dissipating fluorescein at the TE side. The Alexa Fluor 488 is stacked in the ITP window, visualised by the bright zone. Citric acid acts as a non-fluorescent spacer between Alexa Fluor 488 and the TE containing the dissipating fluorescein and cannot be observed by fluorescence microscopy.

The fluorescence intensity was quantified using a PMT using the microscope stage to move the chip. Fig. 5C shows the fluorescence intensity measured using a PMT as a function of the scan time. A narrow peak with high intensity is recorded for Alexa Fluor 488, indicating the ITP window passes the detection spot. Further down in the terminator, a broad zone of lower intensity corresponds to the fluorescein dissipating ion the TE. Regular drops in the fluorescence intensity are caused by the pillars used to support the microfluidic chamber, passing across the detection spot.

\section{FFITP-ESI-MS}

With optimised ITP conditions and the ability to hydrodynamically scan the ITP window past outlet 13, the FFITP was 
(A)

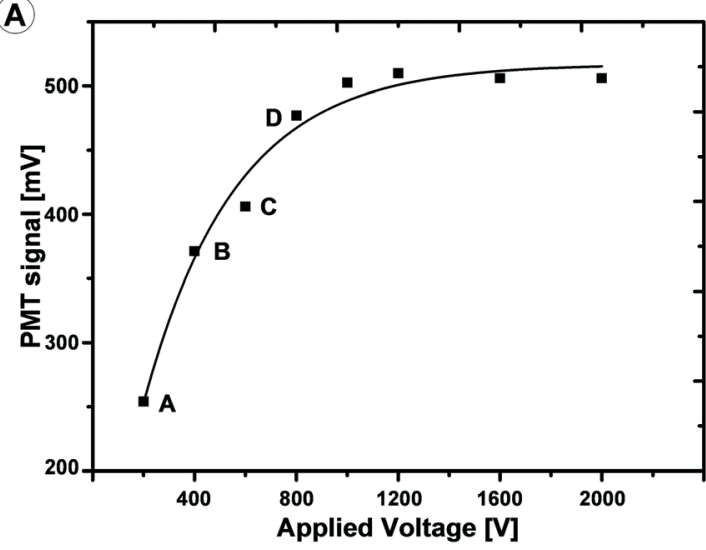

C)

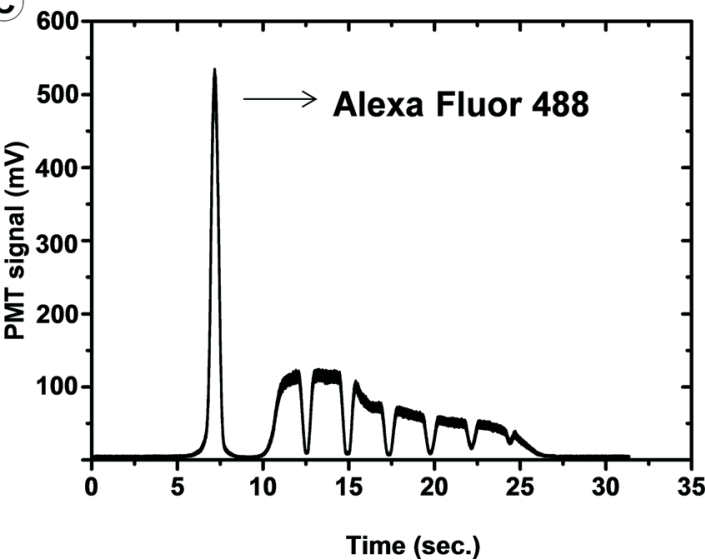

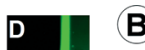

B
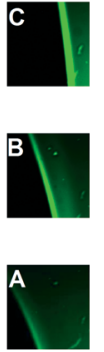

Alexa fluor 488

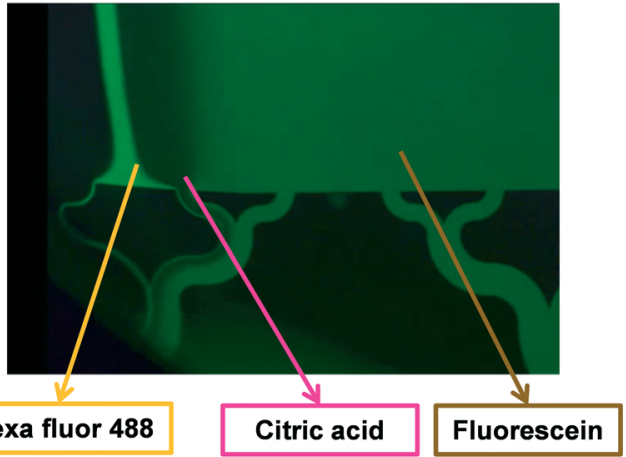

(D)

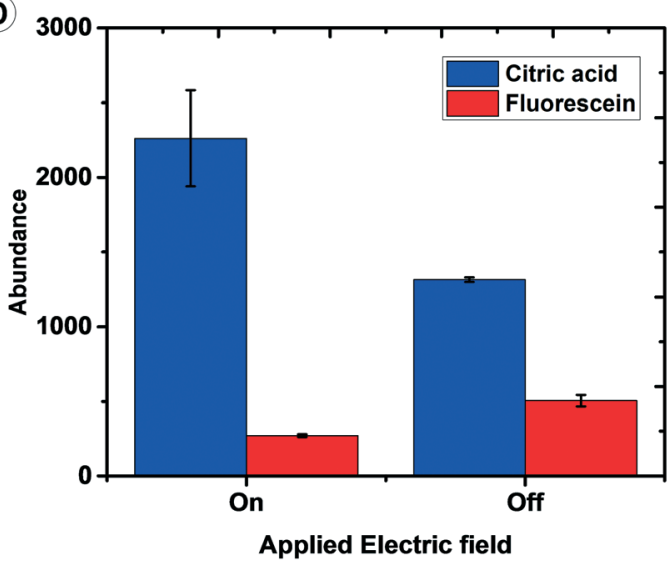

Fig. 5 (A) PMT output signal as function of applied separation voltage with a constant total flow rate of $10 \mu \mathrm{L} \mathrm{min}{ }^{-1}$. The intensity of Alexa Fluor 488 increases by applying a higher voltage and stabilises at $1200 \mathrm{~V}$, indicating the plateau concentration has been reached. (B) Microscope image of the FFITP device which showing the focusing of Alexa Fluor into a sharp band and dissipating fluorescein. (C) PMT signal output which showing the intensity of Alexa Fluor and fluorescein. (D) Continuous data collection was done for more than 50 minutes, while turning on and off the electric field. By applying an electric field the relative abundance in the MS signal for citric acid was increased from 1250 to 2300 , and the abundance of fluorescein was decreased from 500 to 280 .

online connected to the ESI-MS. The simulations and experimental results presented in Fig. 5B and $\mathrm{C}$ indicate that interfering compounds can be removed from the target analytes. This was demonstrated using a sample containing $1 \mathrm{mM}$ fluorescein as interference in a sample containing $1 \mathrm{mM}$ citric acid as target analyte and $1 \mathrm{mM}$ Alexa Fluor 488 as an optical marker enabling fluidic guidance of the ITP zone into the MS (Alexa Fluor 488 does not yield an MS response). In absence of an electric field in the ITP device, the sample flow is directed to the MS, yielding a response for citric acid $(m / z=191)$ and fluorescein $(m / z=331)$. The minimal flow rate through the capillary connecting the FFITP device with the MS to establish a stable electrospray was determined to be $1 \mu \mathrm{L} \mathrm{min}{ }^{-1}$. This required a total flow rate through the chip of $10 \mu \mathrm{L} \mathrm{min}^{-1}$, and results in a delayed detection response by 2 minutes. At this flow rate, $1200 \mathrm{~V}$ was applied over the separation chamber. As the ITP separation takes place in the electric field applied perpendicular to the flow direction, the separation and detection timeframe have been decoupled, hence the composition of the chip effluent is constant over time, provided the ITP process has reached steady state. The changes to the signals for citric acid and fluorescein were monitored with the MS. In agreement with the previous experiments, a drop in fluorescein signal is observed as it dissipates in the TE, whilst the citric acid increases from concentrates behind the Alexa Fluor 488 . These changes are graphically presented in Fig. 5D, showing that the ratio between citric acid and fluorescein increased by a factor 3.2 by applying an electric field. Please note the concentration of citric acid and fluorescein selected to also yield a MS response without the application of a field, and more significant enhancements are expected when the initial concentration of citric acid is lower (peak mode ITP) and/or when the concentration of fluorescein is higher.

As a final experiment, $1 \mathrm{mM}$ glycolic acid was added to the sample to demonstrate the use of the hydrodynamic control to scan across the ITP window. As calculated in Table 1, glycolic acid is expected to be a little slower than citric acid. Fig. 6A shows the MS data output as a function of the scanning time of this process, moving from the LE to the TE and back. First citric acid is detected, which has a mass to charge ratio $(\mathrm{m} / \mathrm{z})$ of 191 . Subsequently, glycolic acid with $\mathrm{m} / \mathrm{z}$ of 73 can be observed. After reaching the TE at around 7 minutes of scan time, the flows were shifted back to the original 

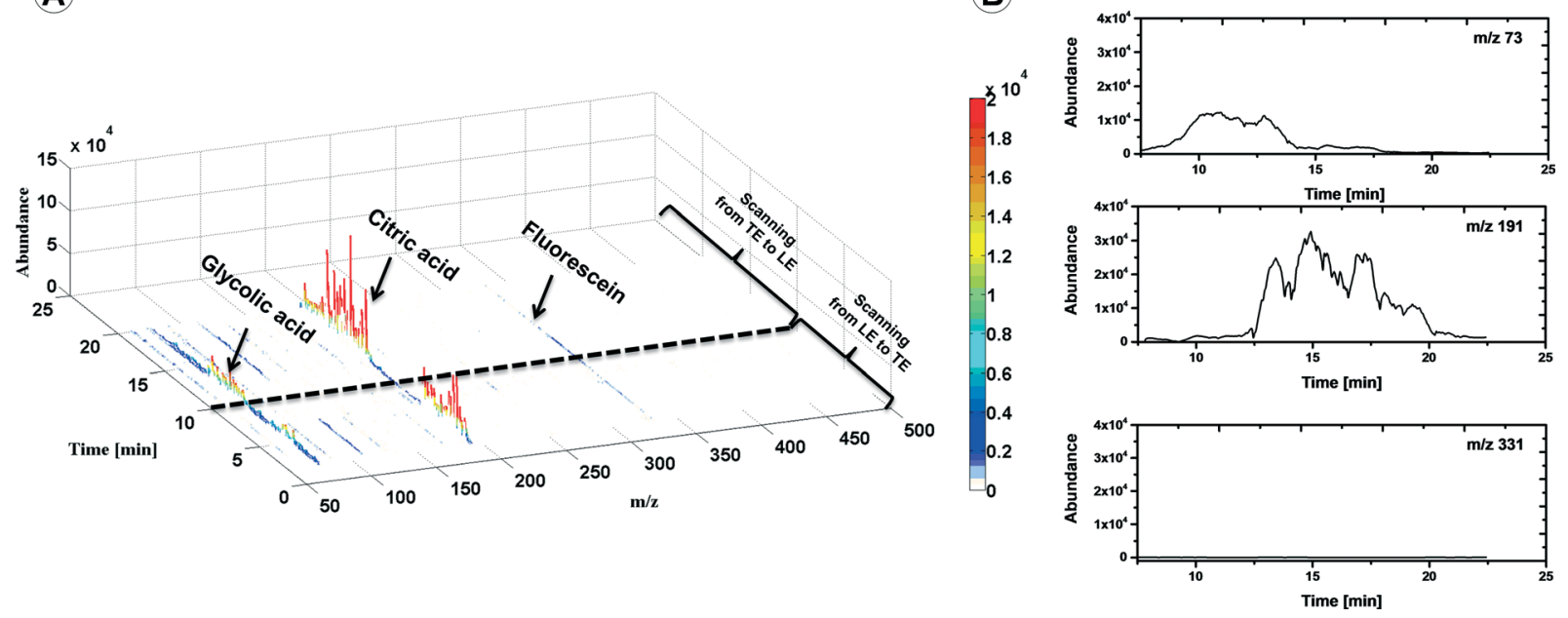

Fig. 6 FFITP-ESI-MS of a sample containing $1 \mathrm{mM}$ each of citric acid, glycolic acid, fluorescein, and Alexa Fluor for optical guidance. Other experimental conditions as in Fig. 5D. (A) Hydrodynamic control allows for scanning of the ITP window from LE to TE (0 to 7 min) and back to LE (7 to $20 \mathrm{~min}$ ). (B) Selected ion isotachopherograms for glycolic acid $(\mathrm{m} / \mathrm{z}=73)$, citric acid $(\mathrm{m} / \mathrm{z}=191)$ and fluorescein $(\mathrm{m} / \mathrm{z}=331)$.

location, showing first glycolic and then citric acid. It is important to realise that fluorescein $(\mathrm{m} / \mathrm{z}=331)$, added as interference, is not detected in the ITP window. The extracted ion isotachopherograms for the three compounds over the period scanning back from TE to LE are given in Fig. 6B. The baseline signal for $m / z=331$ confirms fluorescein has been effectively removed from the ITP window. Fig. $6 \mathrm{~B}$ also demonstrates glycolic and citric acid have been separated into their respective ITP zones, with the slower glycolic acid forming the zone closest to the TE preceding the faster citric acid. It is important to note the absence of citric acid in the glycolic acid zone and vice versa, confirming steady state has been reached.

\section{Conclusions}

The coupling of FFITP with ESI-MS decouples the separation and detection time frame, whilst benefiting from the power of ITP to simultaneously concentrate and purify analytical targets. We have demonstrated the online coupling of FFITPESI-MS by the removal of fluorescein from a set of target analytes. Fluidic scanning of the ITP window was realised by controlling the inlet flows, changing the flow rate ratio supplied to either side of the device. A computational model predicting the shift of the outlet flow as a function of flow rate ratio was experimentally confirmed, enabling a shift of $300 \mu \mathrm{m}$ with $30 \mu \mathrm{m}$ steps.

The connection between the chip and MS was realised using a $100 \mu \mathrm{m}$ ID fused silica capillary, selected to minimize flow resistance and dead volume whilst enabling visual inspection using a fluorescence microscope. For further study where the use of a microscope can be eliminated, shorter capillaries with a narrower ID can be used to allow for lower flow rates and increased residence times in the electric field and hence the time for ITP to establish.
The dissipation of fluorescein into the TE at the same time of the concentration of Alexa Fluor 488 and citric acid to the steady state concentration were recorded using a fluorescence microscope and MS, respectively. The fluorescence intensity across the device is showing a narrow band for the Alexa Fluor 488 in the ITP window, and a broad and less intense signal for the dissipating fluorescein. The changes in abundance for the $\mathrm{m} / \mathrm{z}$ corresponding to citric acid and fluorescein confirmed that the ITP was simultaneously increasing the citric acid concentration to its steady state level and removing the fluorescein. Quantification based on the changes in abundance of the MS signal in presence and absence of an applied electric field demonstrated an increase in the citric acid to fluorescein ratio by a factor of 3.2.

Fluidically scanning across the ITP window past the MS demonstrated the separation of glycolic acid and citric acid by the changes in the abundance at their respective $\mathrm{m} / \mathrm{z}$ ratio. Importantly, no signal was recorded at the $\mathrm{m} / \mathrm{z}$ ratio for fluorescein, demonstrating it was effectively removed from the ITP window.

Based on these encouraging initial results, we are confident that the online coupling of FFITP-ESI-MS will solve problems either where the concentration of the target analytes is very low compared to contaminants, and/or where extended MS studies are required for structure elucidation. Additional engineering of the ESI-MS connection is required to achieve higher spraying stability at low volumetric flow rates. Once solved, it should be possible to apply the technique to biomolecules including peptides and proteins, which may require surface modification of the glass device.

\section{Acknowledgements}

RMG would like to acknowledge the Alexander von Humboldt Foundation for the award of a fellowship. CDMC 
acknowledges FAPESP (2013/06625-2 and 2011/02477-3) for financial support. Furthermore, the authors would like to thank MicroCreate for the fabrication of the device. P. Neužil acknowledges partial financial support by Central European Institute of Technology (CEITEC), grant number CZ.1.05/ 1.1.00/02.0068.

\section{Notes and references}

1 A. R. Fernie, R. N. Trethewey, A. J. Krotzky and L. Willmitzer, Nat. Rev. Mol. Cell Biol., 2004, 5, 763-769.

2 J. W. Jorgenson and K. D. Lukacs, J. Chromatogr., 1981, 218, 209-216.

3 J. W. Jorgenson and K. D. Lukacs, Science, 1983, 222, 266-272.

4 T. P. E. M. Verheggen, J. C. Reijenga and F. M. Everaerts, J. Chromatogr., 1983, 260, 471-477.

5 F. M. Everaerts, J. L. Beckers and T. P. Verheggen, Isotachophoresis: theory, instrumentation and applications, Elsevier, 2011.

6 J. L. Beckers, Electrophoresis, 1995, 16, 1987-1998.

7 J. P. Quirino and S. Terabe, J. Chromatogr., 2000, 902, 119-135.

8 B. Jung, R. Bharadwaj and J. G. Santiago, Anal. Chem., 2006, 78, 2319-2327.

9 D. Bottenus, T. Z. Jubery, Y. Ouyang, W.-J. Dong, P. Dutta and C. F. Ivory, Lab Chip, 2011, 11, 890-898.

10 P. Smejkal, D. Bottenus, M. C. Breadmore, R. M. Guijt, C. F. Ivory, F. Foret and M. Macka, Electrophoresis, 2013, 34, 1493-1509.

11 K. Hannig, Electrophoresis, 1982, 3, 235-243.

12 Y. Xu, C.-X. Zhang, D. Janasek and A. Manz, Lab Chip, 2003, 3, 224-227.

13 E. R. Castro and A. Manz, J. Chromatogr., 2015, 1382, 66-85.
14 S. Jezierski, D. Belder and S. Nagl, Chem. Commun., 2013, 49, 904-906.

15 C.-X. Zhang and A. Manz, Anal. Chem., 2003, 75, 5759-5766.

16 B. R. Fonslow and M. T. Bowser, Anal. Chem., 2005, 77, 5706-5710.

17 D. E. Raymond, Anal. Chem., 1994, 66, 2858-2865.

18 L. Křivánková and P. Boček, Electrophoresis, 1998, 19, 1064-1074.

19 D. Kohlheyer, Anal. Chem., 2008, 80, 4111-4118.

20 D. Kohlheyer, J. C. T. Eijkel, A. van den Berg and R. B. M. Schasfoort, Electrophoresis, 2008, 29, 977-993.

21 S. Kohler, C. Benz, H. Becker, E. Beckert, V. Beushausen and D. Belder, RSC Adv., 2012, 2, 520-525.

22 C. Benz, M. Boomhoff, J. Appun, C. Schneider and D. Belder, Angew. Chem., Int. Ed., 2015, 54, 2766-2770.

23 A. Chartogne, U. R. Tjaden and J. Van der Greef, Rapid Commun. Mass Spectrom., 2000, 14, 1269-1274.

24 T. K. Khurana and J. G. Santiago, Anal. Chem., 2008, 80, 6300-6307.

25 D. Janasek, M. Schilling, J. Franzke and A. Manz, Anal. Chem., 2006, 78, 3815-3819.

26 J. Park, P. Neužil and A. Manz, Presented in part at the Miniaturized Systems for Chemistry and Life Sciences, San Antonio, Texas, USA, 2014.

27 D. Kohlheyer, G. A. J. Besselink, S. Schlautmann and R. B. M. Schasfoort, Lab Chip, 2006, 6, 374-380.

28 F. Hecht, O. Pironneau, A. Le Hyaric and K. Ohtsuka, Product Manual, 2005.

29 P. Gebauer, Z. Malá and P. Boček, Electrophoresis, 2013, 34, 3245-3251.

30 D. Milanova, R. D. Chambers, S. S. Bahga and J. G. Santiago, Electrophoresis, 2011, 32, 3286-3294.

31 V. Hruška, M. Jaroš and B. Gaš, Electrophoresis, 2006, 27, 984-991. 